\title{
Influence of disorder on the perpendicular magnetoresistance of magnetic multilayers
}

\author{
P. Bruno \\ Max-Planck-Institut für Mikrostrukturphysik, Weinberg 2, D-06120 Halle, Germany \\ H. Itoh, J. Inoue, and S. Nonoyama \\ Department of Applied Physics, Nagoya University, Nagoya 464-01, Japan
}

(14 August 1998)

\begin{abstract}
The effect of disorder on the perpendicular magnetoresistance of magnetic multilayers is investigated theoretically. Various kinds of disorder are considered: (i) interface substitutional disorder and (ii) bulk disorder in the various layers and in the leads. The calculations are based upon the non-equilibrium Green's function formalism, together with the recursion method for calculating the real-space Green's function.
\end{abstract}

published in: J. Magn. Magn. Mater. 198-199, 46-48 (1999).

\section{INTRODUCTION}

The common wisdom about giant magnetoresistance (GMR) is that it is due to spin dependent scattering.t. Although the transport is certainly diffusive for most systems investigated experimentally, it has been pointed out that pure ballistic transport as well would lead to GMR for the perpendicular current geometry. 2 Subsequently, it was proposed that for ballistic perpendicular transport, the electron confinement within the layers would yield a quantum size effect, i.e., an oscillatory behavior of the confyctance and of the GMR as a function of layer thickness. 3 ,

In the present paper, we investigate the influence of disorder on perpendicular magneto-resistance, and discuss the crossover from ballistic to diffusive transport as disorder increases.

\section{MODEL}

The model considered here consists of two ferromagnetic layers of thickness $L$ separated by a non-magnetic spacer layer of thickness $D$; the structure is sandwiched between two ideal leads connected to ideal reservoirs. We use a simple-cubic single-band tight-binding Hamiltonian with a nearest-neighbor hopping. The hopping parameter, $t$, is the same for all pairs of nearest neighbors (for numerical calculations, $t=-1$ will be assumed). The onsite energy of site $i, \varepsilon_{i}$, depends on the chemical nature of the site and (for magnetic sites) on its spin. The majority spin on-site energy of the magnetic sites is taken equal to the on-site energy of nonmagnetic sites, and the minority spin on-site energy of the magnetic sites is $0.3|t|$ higher than the latter. The Fermi level is taken $0.8|t|$ above the bottom of the band; this corresponds to a quasi-freeelectron case, with quasi-parabolic band structure and quasi-spherical Fermi surface.

Two kinds of models for disorder are considered: (i) random substitutional disorder, and (ii) the Anderson model of disorder. Model (i) is particularly well suited to study the effect of interface interdiffusion. In model (ii), a random on-site energy (characterized by a square distribution of width $W$ ) is added to the on-site energy of the perfect case; this is a convenient model for bulk disorder. For both cases, a periodic (along the in-plane directions) model of disorder with $10 \times 10$ supercell is considered. We did not perform systematically an average over disorder configurations, but we checked that this 100-sites supercell is large enough for fluctuations to be unimportant. For $\mathbf{k}_{\|}$summations, we take $100 \mathbf{k}_{\|}$-points in the two-dimensional Brillouin zone corresponding to the supercell; this sampling is equivalent to taking $10^{4}$ $\mathbf{k}_{\|}$-points for the perfect case.

\section{THEORY}

The calculation of the conductance of the structure is based upon the non-equilibrium Green's function formalism.5 When applied to the case of a two-terminal ballistic mesoscopic conduftor we obtain the following result for the conductance: 6 -

$$
G=\frac{e^{2}}{h} \operatorname{Tr}_{\mathbf{k}_{\|}, \sigma}\left(\Gamma_{l} G_{l r}^{R} \Gamma_{r} G_{r l}^{A}\right)
$$

In the above equation, the upper indices $R$ and $A$ refer to the retarded and advanced Green's function, respectively; the lower $l$ and $r$ indices refer to the left and right reservoirs, respectively. Thus, $G_{l r}^{R}$ is the offdiagonal retarded Green's function linking the left reservoir to the right reservoir; $\Gamma_{l}$ (respectively, $\Gamma_{r}$ ) are given by $\Gamma_{l(r)}=t^{2} A_{l(r)}$, where $A_{l}$ (respectively, $A_{r}$ ) is the spectral density in the first plane of the left (respectively, right) reservoir, when it is decoupled from the conductor. All the quantities in Eq. (3.1) are taken at the Fermi level. The conductance obtained here from the non-equilibrium Green's function formalism, Eq. (3.1), can be shown to be equivalent to the ones obtained from the $K_{b}$ bo formula, and from the Landauer-Büttiker formalism. 
By separating the $\mathbf{k}_{\|}$-conserving and $\mathbf{k}_{\|}$-nonfusive contributions to the total conductance: conserving terms, we can separate the ballistic and dif-

$$
\begin{aligned}
G_{\text {bal }} & \equiv \frac{e^{2}}{h} \sum_{\mathbf{k}_{\|}} \operatorname{Tr}_{\sigma}\left[\Gamma_{l}\left(\mathbf{k}_{\|}\right) G_{l r}^{R}\left(\mathbf{k}_{\|}, \mathbf{k}_{\|}\right) \Gamma_{r}\left(\mathbf{k}_{\|}\right) G_{r l}^{A}\left(\mathbf{k}_{\|}, \mathbf{k}_{\|}\right)\right] \\
G_{\text {dif }} & \equiv \frac{e^{2}}{h} \sum_{\mathbf{k}_{\|} \neq \mathbf{k}_{\|}^{\prime}} \operatorname{Tr}_{\sigma}\left[\Gamma_{l}\left(\mathbf{k}_{\|}\right) G_{l r}^{R}\left(\mathbf{k}_{\|}, \mathbf{k}_{\|}^{\prime}\right) \Gamma_{r}\left(\mathbf{k}_{\|}^{\prime}\right) G_{r l}^{A}\left(\mathbf{k}_{\|}^{\prime}, \mathbf{k}_{\|}\right)\right] .
\end{aligned}
$$

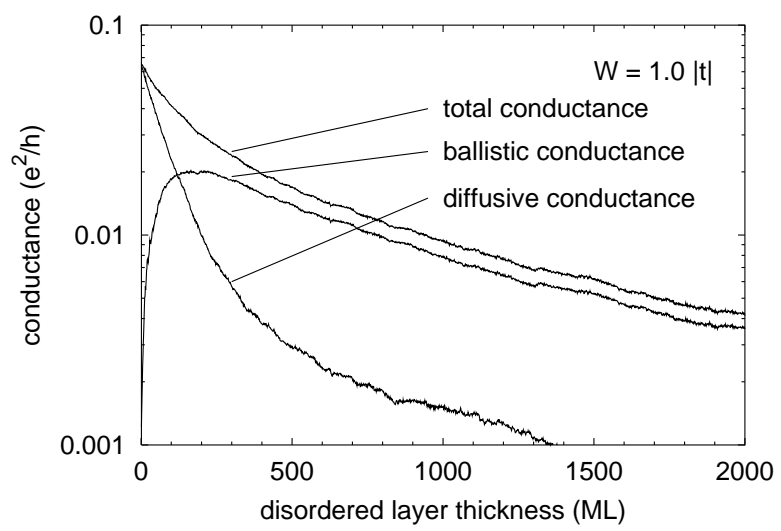

FIG. 1. Conductance of a disordered nonmagnetic layer as a function of layer thickness.

\section{RESULTS AND DISCUSSION}

Before considering the problem of perpendicular magnetoresistance, we illustrate our method by presenting the conductance of a nonmagnetic layer of thickness up to 2000 monolayers (ML), with an Anderson-like disorder of width $W=1.0|t|$. The results are shown in Fig. 11. For small thicknesses, the transport is quasi-ballistic, but for thicknesses larger than the mean-free path (approximately 400 ML here), the transport becomes diffusive.

Let us now consider the problem of the perpendicular magneto-resistance. We define the magnetoresistance as 8 $A \equiv\left(G_{F}-G_{A F}\right) /\left(G_{F}+G_{A F}\right)$. The results are displayed in Fig. 2. The magneto-resistance of the perfect system (Fig. 2a) exhibits the oscillations due to the quantum size effect. We have investigated the case of interface interdiffusion (Fig. 2p) by including two planes of random substitutional alloy: it appears that the latter influences only weakly the magnetoresistance. We have also performed calculations treating the interface alloy within the virtual crystal approximation: the magnetoresistance (not shown here) is almost identical to the "exact" one (Fig. 2b); the effect of is interface interdiffusion is thus merely to replace the abrupt interface by a graded in- terface, which reduces the reflectivity, but produces little diffuse scattering.

If we include bulk disorder in the spacer (Anderson model of disorder) the diffuse scattering leads to reduction of the magneto-resistance for large spacer thickness, but the quantum size effect is still present (Fig. 22c).

Next we simulate the effect of disoder in the leads by including a rather thick (400 ML) disordered region (Anderson model of disorder) in the left leade (Fig. 2 d ). This yields a significant reduction of magneto-resistance: this is easily explained as the effect of an additional resistance in series with the multilayer. The other effect is that the quantum size effect is strongly perturbed.

In order to understand better the effect of disorder on the quantum size effect, we show in Fig. 3 the minorityspin contribution to the conductance of the ferromagnetic alignment (which is the one giving rise to the quantum size effect here). We see that, for a disordered lead (Fig 3 d ), the amplitude of the conductance oscillation is reduced by a factor of 4 , while the conductance itself is only reduced by 30 percent. Thus, this suggests that diffuse scattering in the leads supresses the quantum size effect (here it is not completely supressed because the disordered region is rather thin and the ballistic conductance still accounts for 70 percent of the total conductance).

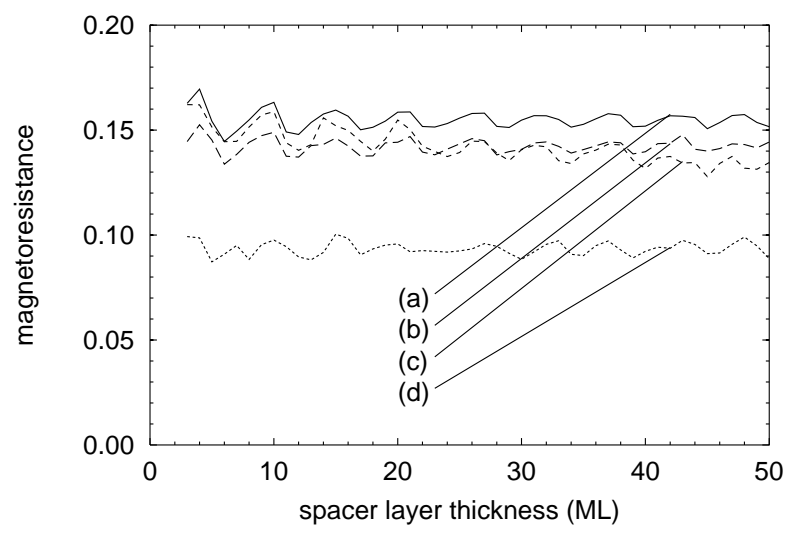


FIG. 2. Magneto-resistance. (a) perfect case; (b) interface interdiffusion (2 layers of substitutional disorder); (c) bulk disorder in spacer layer (Anderson model, $W=0.5|t|$ ); (d) 400 disordered layers in left lead (Anderson model, $W=0.5|t|$.

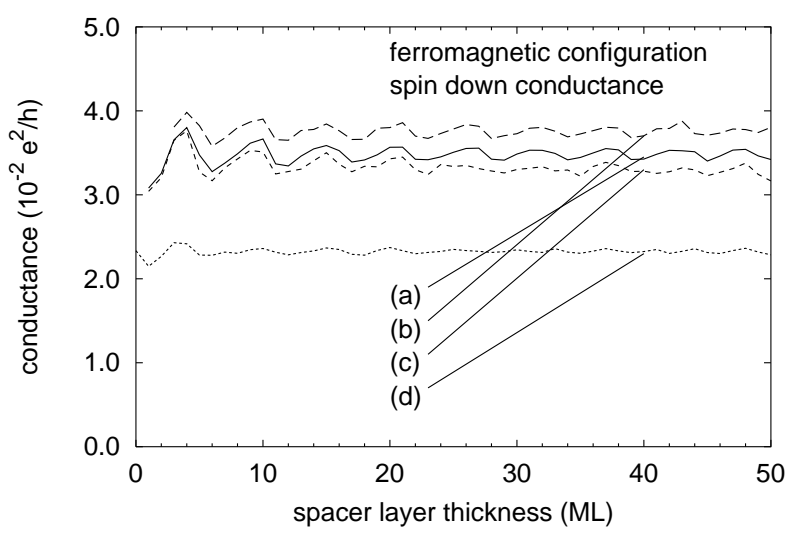

FIG. 3. Minority-spin conductance for the ferromagnetic configuration. (a)-(d): same as Fig. 2 .

\section{ACKNOWLEDGEMENTS}

P.B. gratefully thanks the Japan Society for the Promotion of Science for the financial support of his stay in
Nagoya during which this work was initiated.

${ }^{1}$ M.N. Baibich, J.M. Broto, A. Fert, F. Nguyen Van Dau, F. Petroff, P. Etienne, G. Creuzet, A. Friederich, and J. Chazelas, Phys. Rev. Lett. 61, 2472 (1988).

${ }^{2}$ K.M. Schep, P.J. Kelly, and G.E.W. Bauer, Phys. Rev. Lett. 74586 (1995).

${ }^{3}$ J. Barnas and Y. Bruynseraede, Europhys. Lett. 32, 167 (1995).

${ }^{4}$ J. Mathon, M. Villeret, and H. Itoh, Phys. Rev. B 52, R6983 (1995).

${ }^{5}$ L.P. Kadanoff and G. Baym, Quantum Statistical Mechanics, Benjamin, New York (1962); L.V. Keldysh, Soviet Phys. JETP 20, 1018 (1965).

${ }^{6}$ S. Datta, J. Phys.: Condens. Matter 2, 8023 (1990).

${ }^{7}$ P. Bruno, unpublished.

${ }^{8}$ Note that the present definition differs from the one used by other authors, who normalize the magneto-resistance by $G_{F}$ instead of $G_{F}+G_{A F}$ as done here; the present convention gives values strictly bound between -1 and +1 , and gives numerical values that are smaller by typically a factor 2 .

${ }^{9}$ For technical reasons, disorder could not be included conveniently for both leads, but results with 2 disordered leads may be expected to be similar to the one obtained with 1 disordered lead. 